\title{
ANÁLISE DA RELAÇÃO ENTRE INCIDÊNCIA E SEVERIDADE FOLIAR DA CERCOSPORIOSE DA BETERRABA
}

\section{Leandro Luiz Marcuzzo $^{1 *}$, Sheila Chaiana Harbs ${ }^{1}$, Bruna Kotkoski $^{1}$, Aline Cristina Paulakoski ${ }^{1}$}

\author{
${ }^{1}$ Instituto Federal Catarinense, Campus Rio do Sul, Rio do Sul, 89163-356, Santa Catarina, Brasil
}

*Autor para correspondência: Leandro Luiz Marcuzzo; e-mail: leandro.marcuzzo@ifc.edu.br

Recebido: 2206/2020, Aceito: 3009/2021, Publicado: 18/10/2021

\begin{abstract}
Resumo
A ocorrência da cercosporiose da beterraba pode reduzir o rendimento e comprometer a sua qualidade. O objetivo do trabalho foi analisar a relação entre incidência e severidade na intensidade da cercosporiose da beterraba. $\mathrm{O}$ experimento foi conduzido com o cultivar Boro no Instituto Federal Catarinense/Campus Rio do Sul. O delineamento foi de blocos casualizados, com quatro repetições e cinco tratamentos constituídos de pulverização com fungicida conforme o sistema de previsão proposto por Marcuzzo et al. com valores de severidade estimada (SE) acumulado de 0,15; 0,25 e 0,35 em comparação a aplicação a cada 5 e 7 dias com objetivo de gerar um gradiente de intensidade de doença nos anos de 2018 e 2019. Semanalmente foi avaliada a intensidade da doença pela severidade e incidência. Os dados obtidos foram submetidos à análise de regressão linear. Utilizando a equação com melhor ajuste nas duas safras e substituindo o valor de severidade de $1 \%$ obtém-se incidência de $4,8 \%$. A severidade estimada com base na incidência e vice-versa possibilita seu uso adequado pela assistência técnica no monitoramento da cercosporiose da beterraba.
\end{abstract}

Palavras-chave: Beta vulgaris ssp. vulgaris, Cercospora beticola, intensidade de doença, epidemiologia, monitoramento.

\section{RELATION ANALYSIS BETWEEN INCIDENCE AND SEVERITY IN CERCOSPORA LEAF SPOT IN BEET}

\begin{abstract}
The occurrence of Cercospora leaf spot in beet can reduce the productivity and compromise the quality. The objective was to evaluate the relation between incidence and severity intensity of Cercospora leaf spot in beet. The experiment was conducted with the cultivar Boro in Catarinense Federal Institute/Campus Rio do Sul. The design was a randomized block design with four replications and six treatments made of fungicide, as the forecasting system proposed by Marcuzzo et al. with estimated severity (ES) accumulated of 0,15;0,25, and 0,35 compared to application every 5 and 7 days in order to promote a disease gradient intensity in the years 2018 and 2019. The intensity of the disease was evaluated weekly using the severity and incidence. The data were submitted to linear regression analysis. Using the equation with the best fit in two seasons and replacing the severity value of $1 \%$ is obtained incidence of $4.8 \%$. The estimated severity based on the incidence and the opposite enable its adequate use for technical assistance in the monitoring of Cercospora leaf spot in beet.
\end{abstract}

Keywords: Beta vulgaris ssp. vulgaris, Cercospora beticola, disease intensity, epidemiology, monitoring, 


\section{Introdução}

A cultura da beterraba vermelha ou de mesa (Beta vulgaris ssp. vulgaris) ocupa destaque entre as hortaliças de maior expressão econômica do Brasil. Além disso, constitui atividade socioeconômica de grande relevância para pequenos produtores da região sul, em especial na região do Alto Vale do Itajaí em Santa Catarina, onde se concentra em torno de 1600 hectares (ha) da cultura (EPAGRI, 2015).

Entretanto, vários fatores contribuem para a baixa produtividade da beterraba, dentre eles, estão às doenças de diversas etiologias, que causam danos à cultura. Tais como a cercosporiose causada por Cercospora beticola Sacc., uma doença de grande importância na região do alto vale do Itajaí, que na época de cultivo encontra condições favoráveis de temperatura $\left(\geq 22^{\circ} \mathrm{C}\right)$ e alta umidade $(\geq 90 \%)$. A doença incide por toda a parte aérea da planta e acaba comprometendo a produtividade da cultura (Ferreira \& Tivelli, 1989; Tivelli et al., 2011).

É importante destacar que os tratamentos fitossanitários utilizados na cultura da beterraba são realizados na maioria das vezes de forma inadequada, sem ciência se há necessidade ou não da aplicação de fungicidas, utilizando normalmente aplicações semanais com base em calendários fixos; essa prática resulta em número excessivo de aplicações e consequente contaminação do ambiente (Dellamatrice, 2000). Segundo Bergamim Filho e Amorim (2011), os sistemas de previsão de doenças em plantas são representações simplificadas da realidade e preveem o início ou o desenvolvimento futuro de uma doença (Barreto et al., 2004; Krause \& Massie, 1975; Reis, 2004), além da previsão do momento adequado à pulverização com fungicidas, considerando que o patógeno se encontra em quantidades suficientes para iniciar uma epidemia e que o hospedeiro seja suscetível (Mizubuti, 1999).

Entre os critérios técnicos que justifica a aplicação de fungicidas tem como base o monitoramento da intensidade da doença que pode ser expresso pela incidência e/ou severidade foliar. Ou seja, o percentual de folhas sintomáticas ou a porcentagem da área da folha afetada pelos sintomas, respectivamente, no caso das doenças foliares (Bergamin Filho \& Amorim, 1996; Vale et al., 2004).

A avaliação da severidade foliar é mais onerosa, porém é a que melhor demonstra a intensidade de manchas foliares. A sua determinação com mais acurácia pode ser feita por estimativas visuais da severidade, com o auxílio de escalas diagramáticas ou por análise de imagem em computador (Azevedo, 1997). Em função da maior subjetividade nas estimativas e necessidade de treinamento ou uso contínuo de uma escala diagramática, o uso da severidade tem sido pouco usado pela assistência técnica, comparado ao da incidência, sendo mais simples, rápida e objetiva.

Com base nisso, o presente trabalho teve como objetivo analisar as relações entre a incidência e severidade e obter equações preditivas da severidade da cercosporiose da beterraba a partir de mensurações de incidência e severidade em função de diferentes gradientes de intensidade da doença.

\section{Material e Métodos}

O trabalho foi conduzido no Instituto Federal Catarinense - IFC/Campus Rio do Sul, no município de Rio do Sul - SC, (Latitude: $27^{\circ} 11^{\prime} 07^{\prime}$ ' S e Longitude: 49 39'39' W, altitude 655 metros acima do nível do mar) durante o período de 14 de setembro a 14 de dezembro de 2018 e de 16 de setembro a 14 de dezembro de 2019 totalizando 13 semanas após a semeadura de cada experimento. Segundo a classificação de Köeppen, o clima local é subtropical úmido (Cfa) e solo classificado como Cambissolo Háplico Tb distrófico (Santos et al., 2018) com os seguintes atributos químicos: $\mathrm{pH}$ em água de 6,0 ; teores de $\mathrm{Ca}^{+2}, \mathrm{Mg}^{+2}, \mathrm{Al}^{+3}$ e CTC de 4,2; 1,8;0,0 e 9,54 cmolc. $\mathrm{dm}^{-3}$, respectivamente; saturação por bases de $66,49 \%$, teor de argila de $30 \%$ e teores de P e K de 14 e $134 \mathrm{mg} \cdot \mathrm{dm}^{-3}$, respectivamente. Os dados meteorológicos foram obtidos de uma estação Davis ${ }^{\circledR}$ Vantage Vue $300 \mathrm{~m}$ localizado ao lado do experimento e os dados médios durante a condução do experimento foram de 18,9 e $19,2^{\circ} \mathrm{C}$ para temperatura do ar, de 14,6 e 14 de umidade relativa do ar $\geq 90 \%$ e a precipitação pluvial acumulada foi de 330 e $446,5 \mathrm{~mm}$ respectivamente para 2018 e 2019.

Sementes de beterraba da cultivar Boro foram semeadas a campo em quatro repetições constituídas de uma área de 1,5 X 1,25 m utilizando cinco linhas com espaçamento de $0,25 \mathrm{~m}$ entre linhas e $10 \mathrm{~cm}$ entre plantas com 40 plantas $/ \mathrm{m}^{2}$ (equivalente a 400.000 plantas.ha ${ }^{-1}$ ), ficando com um estande final de 75 plantas em cada repetição. A calagem, adubação, tratos culturais seguiram as normas da cultura (Tivelli et al., 2011). Não foram utilizados inseticidas devido a não ocorrência de insetos no período de avaliação.

Para que houvesse inóculo na área, mudas de beterraba cv. Boro com 30 dias de idade foram inoculadas com auxílio de um atomizador portátil uma suspensão $\left(10^{4}\right)$ de conídios de C. Beticola obtidos de folhas de beterraba e caracterizado morfologicamente e após 24 horas de câmara úmida com fotoperíodo de 12 horas de luz foram transplantadas a cada um metro linear ao redor do experimento no dia da semeadura. 
Para o controle da cercosporiose foram comparados os seguintes regimes de pulverização com mancozeb $(80 \%)+$ oxicloreto de cobre (50\%) na dose de $250 \mathrm{~g}+$ $200 \mathrm{~g} \mathrm{pc}^{-1}{ }^{-1}$ baseado no modelo descrito por Marcuzzo et al. (2016) expresso em SE $=0,0001105 *(((x-$ $\left.\left.8)^{2,294387}\right) *\left((36-x)^{0,955017}\right)\right) *(0,39219 /(1+25,93072 *$ exp $\left.\left.\left(-0,16704^{*} y\right)\right)\right)$, onde SE, representa o valor da severidade estimada ( 0,1 ou seja, percentual da doença /100); $x$, a temperatura $\left({ }^{\circ} \mathrm{C}\right)$ e $y$, o molhamento foliar (horas) avaliado pelas horas de umidade relativa $\geq 90 \%$.

Os tratamentos foram constituídos de valores de SE de 0,$15 ; 0,25$ e 0,35 e comparados com sistema convencional com pulverização a cada 5 e 7 dias. A pulverização no sistema de previsão foi realizada quando o somatório diário dos valores de $\operatorname{SE}(15,25$, 35) foi atingida, sendo então zerado o somatório e iniciada nova soma dos SE. A cada ocorrência de 25 $\mathrm{mm}$ de chuva, todos os tratamentos eram pulverizados, zerados e reiniciava-se a contagem do somatório dos valores de severidade.

As pulverizações iniciaram-se a partir dos 21 dias após a semeadura e foram efetuadas com um pulverizador costal eletrônico Jetbras ${ }^{\circledR}$ calibrado para 400 L.ha $^{-1}$, a severidade da doença foi avaliada através de escala diagramática proposta por May de Mio et al. (2008) em cada folha presente na planta e a incidência foi avaliada pelas folhas totais em relação às folhas doentes. Os diferentes programas de pulverização tiveram como objetivo gerar um gradiente de intensidade de doença conforme metodologia proposta por Sah \& Mackenzie (1987), para determinar em qual severidade é correspondente a incidência e vice-versa., onde os dados obtidos foram submetidos a análise de regressão pelo programa estatístico Microsoft Office Excel versão 2007, a fim de obter equações preditivas da severidade em função da incidência para avaliações em cada estádio de desenvolvimento da cultura e em cada safra agrícola.

\section{Resultados e discussão}

Os dados obtidos em diferentes tratamentos de pulverização baseado no sistema de Marcuzzo et al. (2016) em relação ao sistema convencional de 5 e 7 dias quando comparados com a testemunha se ajustaram a equação linear, permitindo relacionar as variáveis testadas durante o desenvolvimento da doença (Fig. 1). Os coeficientes angulares foram significativos, resultados demonstrados pela inclinação da reta, o que possibilita relacionar a incidência e a severidade foliar da cercosporiose da beterraba e ao analisar os coeficientes de determinação, observa-se que os menores valores ocorreram na safra 2019, o que pode ser atribuído à menor severidade da doença, pois a baixa severidade dificulta a obtenção de uma boa acurácia e precisão na mensuração das doenças, uma vez que as lesões iniciais são pequenas e variáveis em forma, cor e presença ou não de tecido necrótico.

Os dados foram melhores ajustados pela severidade $=2,888 \mathrm{I}-12,81\left(\mathrm{R}^{2}=0,936\right)$ em sete dias na safra de 2018 (Fig. 1). Esse mesmo tratamento em 2019 teve o melhor ajuste quando comparado aos demais regimes de pulverização com coeficiente de determinação de 0,841 e ajustado a severidade de 8,750I-70,11.

Utilizando a equação com melhor ajuste $\mathrm{y}=2,88 \mathrm{I}-$ $12,81\left(\mathrm{R}^{2}=0,936\right)$ que ocorreu em sete dias (Fig. 1) e fazendo uma simulação utilizando valor arbitrário de $1 \%$ de severidade na planta obtém-se incidência de $4,8 \%$. Como a severidade está relacionada ao programa de pulverização, o grau de relação entre a incidência e a severidade foliar variou em função de que a ocorrência da doença se deu praticamente sobre todas as folhas da planta, enquanto a severidade ainda era muito variável.

Nas fases iniciais de uma epidemia de manchas foliares a incidência e severidade aumentam até que todas as folhas sejam infectadas, a partir desse ponto, o aumento da intensidade da doença pode ocorrer somente pela severidade (James \& Shih, 1973). A incidência é útil para avaliar doenças quando a epidemia se encontra em sua fase inicial podendo nesse caso, ser correlacionada com a severidade (Amorim, 1995). Essa mesma técnica foi utilizada por Marcuzzo e Carvalho (2017) para avaliar a relação entre a incidência e severidade do míldio da cebola e também para a queima das pontas das folhas da cebola (Marcuzzo, Kotkoski e Wernke, 2019), demonstrando valores significativos entre as variáveis com intuito de facilitar a avaliação da doença nessa cultura. 


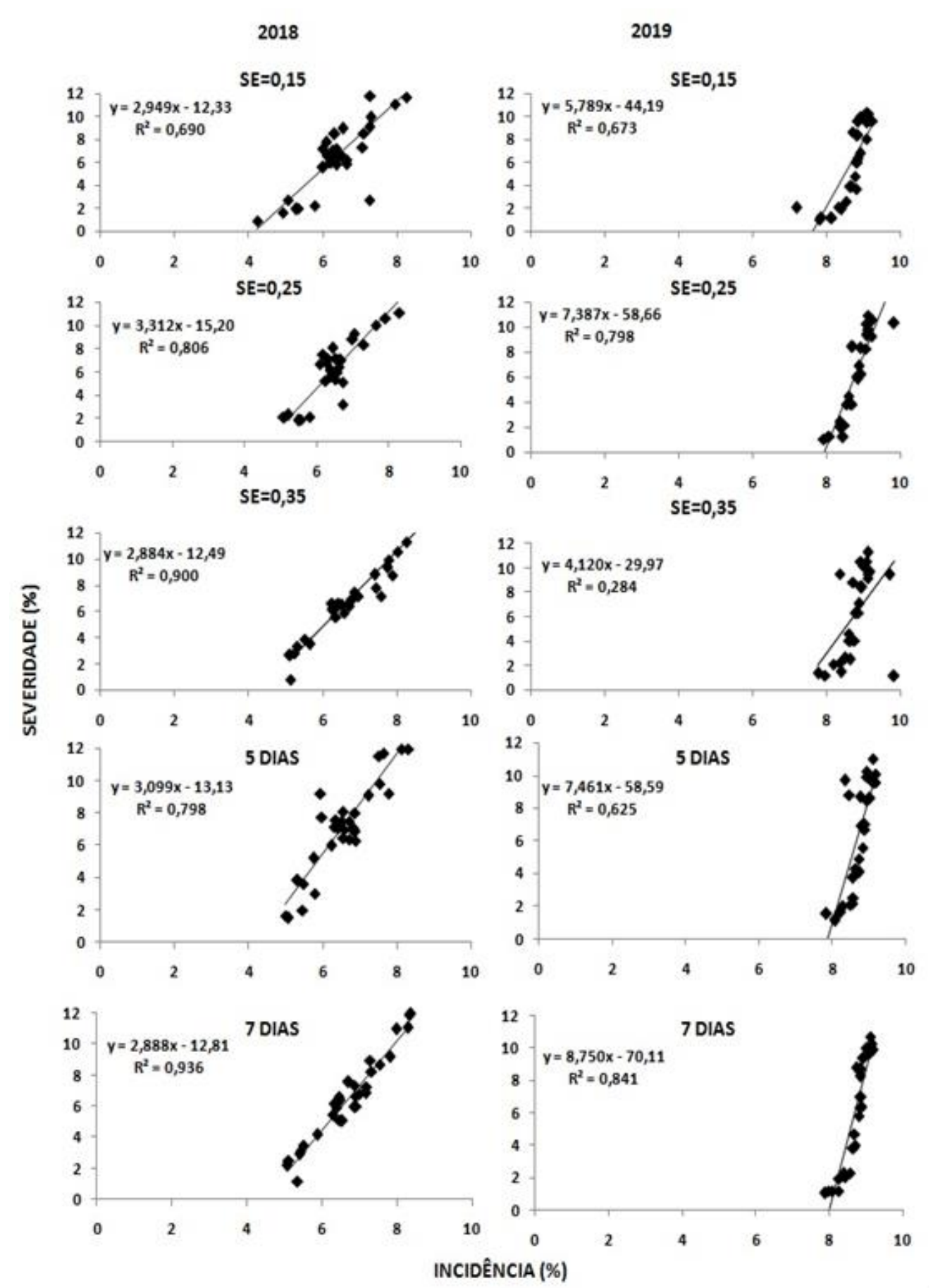

Figura 1. Relação entre a incidência e a severidade foliar da cercosporiose (Cercospora beticola) da beterraba em diferentes sistemas de pulverização nas safras 2018 e 2019 em Rio do Sul, SC.

\section{Considerações finais}

Plântulas de $S$. parahyba tem emergência, crescimento inicial e massa seca maiores quando produzidas com maior volume de substrato a partir de sementes grandes. Apesar disso, outros estudos se tornam necessários, a partir da associação entre dispersão das sementes no campo e a viabilidade.

\section{Agradecimentos}

Ao CNPq e ao IFC/Campus Rio do Sul pela concessão de bolsa de iniciação científica.

\section{Referências}

Amorim, L. Avaliação de doenças. (1995). In: Bergamim Filho, A.; Kimati, H.; Amorim, L., (Orgs). 
Manual de fitopatologia. (3a ed., v.1, Cap.31, pp.234235). São Paulo: Ceres.

Azevedo, L.A.S. (1997). Manual de quantificação de doenças de plantas. São Paulo: Novartis Biociências.

Barreto, M.; Vale, F.X.R.; Paul, P.A.; Scaloppi, E.A.G.; Andrade, D.F.A.A. (2004). Sistemas de previsão e estação de aviso. In: Vale, F.X.R.; Jesus Junior, W.C.; Zambolim, L. (Orgs.). Epidemiologia aplicada ao manejo de doenças de plantas. (Cap. 6, pp.243-266). Belo Horizonte: Perffil,

Bergamim Filho, A.; Amorim L. (2011). Sistemas de previsão e avisos. In: Amorim, L.; Rezende, J.A.M.; Bergamim Filho, A. (Orgs.). Manual de fitopatologia. (4a ed. v.2, Cap. 18, pp. 389-408). São Paulo: Ceres.

Bergamin Filho, A.; Amorim, L. (1996). Doenças de plantas tropicais: epidemiologia e controle econômico. São Paulo: Ceres.

Dellamatrice, P.M. (2000). Degradação do herbicida 14C Diuron por Acinetobacter baumannii e pela microbiota do solo. Dissertação (Mestrado em Ciências) - Energia nuclear na agricultura, Universidade de São Paulo, Piracicaba.

EPAGRI.- Empresa catarinense de pesquisa e extensão rural de Santa Catarina. 2015. Levantamento de espécies de hortaliças cultivadas na região do Alto Vale do Itajaí, 2010 e 2014. (7p. Relatório de pesquisa). Epagri: Ituporanga.

Ferreira, M.D.; Tivelli, S.W. (1989). Cultura da beterraba: recomendações gerais. (14p, Boletim Técnico, 2). Guaxupé: Cooxupé.

James, W. C.; Shih, C. S. (1973). Relationship between incidence and severity of powdery mildew and leaf rust on winter wheat. Phytopathology, 63, 183-187.

Krause, R.A.; Massie, L.B. (1975). Predictive systems: modern approaches to disease control. Annual Review of Phytopathology, 13, 31-47.

Marcuzzo, L.L.; Kotkoski, B.; Wernke, C. (2019). Relações lineares entre incidência e severidade foliar da queima das pontas das folhas da cebola. Summa Phytopathologica, 45, 107-110.

Marcuzzo, L.L.; Carvalho, J. (2017). Relações lineares entre incidência e severidade foliar do míldio da cebola. Summa Phytopathologica, 43, 344-347.
Marcuzzo, L.L.; Haveroth, R.; Nascimento, A. (2016). Influence of temperature and leaf wetness duration on the severity of Cercospora leaf spot of beet. Summa Phytopathologica, 42, 89-91.

May de Mio, L.L.; Oliveira, R.A.; Floriani, A.M.V.; Schuber, J.M.; Poltronieri, A.S.; Araujo, M.A.; Tratch, R. (20080. Proposta de escala diagramática para quantificação da cercosporiose da beterraba. Scientia Agraria, 9, 331-337.

Mizubuti, E.S.G. (1996). Sistema de previsão de doenças de plantas: uma ferramenta útil? In: Zambolim L. (Org.) $1^{\circ}$ Encontro de manejo integrado de doença e pragas. (pp.42-46). Viçosa: Universidade Federal de Viçosa.

Reis, E.M. (2004). Previsão de doenças de plantas. Passo Fundo: Universidade de Passo Fundo.

Sah, D.N.; Mackenzie, D.R. (1987). Methods of generating different levels of disease epidemics in loss experiments. In: Teng, P.S. (Org.). Crop loss assessement and pest management. (pp.90-95). St Paul: Americam phytopathological Society.

Santos, H.G.; Jacomine, P.K.T.; Anjos, L.H.C.; Oliveira, V.Á.; Lumbreras, J. F.; Coelho, M.R.; Almeida, J.A.; Araujo Filho, J.C.; Oliveira, J.B.; Cunha, T. J.F. (2018). Sistema Brasileiro de classificação de solos. (5a ed.) Brasília, DF: Embrapa. Disponível em: <https://www.embrapa.br/busca-de-publicacoes//publicacao/1094003/sistema-brasileiro-declassificacao-de-solos >. Acesso em 15 jun 2021.

Tivelli, S.W; Factor, T.L.; Teramoto, J.R.S.; Fahi, E.G.; Moraes, A.R.A.; Trani, P.E.; May, A. 2011 Beterraba, do plantio à comercialização. (Série Tecnologia APTA. Boletim Técnico IAC, 210). Campinas: Instituto Agronômico.

Vale, F.X.R.; Jesus Junior, W.; Zambolim, L. (2004). Epidemiologia aplicada ao manejo de doenças de plantas. Belo Horizonte: Perffil. 